\title{
Myocarditis associated with Covid-19 disease: a systematic review of published Case reports and Case series
}

\author{
Sawai Singh Rathore ${ }^{1}$, Gianpier Alonzo Rojas ${ }^{2}$, Manush Sondhi ${ }^{3}$, Suveenkrishna Pothuru ${ }^{4}$, \\ Reshma Pydi ${ }^{5}$, Neeraj Kancherla ${ }^{5}$, Romil Singh ${ }^{6}$, Noman Khurshid Ahmed ${ }^{7}$, Jill Shah ${ }^{8}$, \\ Sohaib Tousif ${ }^{9}$, Unaiza Tariq Baloch ${ }^{10}$, and Qingqing Wen ${ }^{11}$ \\ ${ }^{1}$ Dr Sampurnanand Medical College \\ ${ }^{2}$ School of medicine,Universidad Peruana Cayetano Heredia, Lima \\ ${ }^{3}$ Kasturba Medical College Manipal \\ ${ }^{4}$ Ascension Via Christi Hospital in Manhattan \\ ${ }^{5}$ Andhra Medical College \\ ${ }^{6}$ Mayo Clinic Rochester \\ ${ }^{7}$ Dow Medical College \\ ${ }^{8}$ Tambov state University named after G.R.Derzhavin \\ ${ }^{9}$ Ziauddin Medical University \\ ${ }^{10}$ The Aga Khan University Hospital \\ ${ }^{11}$ University of California Los Angeles, Fielding School of Public Health,
}

February 1, 2021

\begin{abstract}
Background: Covid-19 is an extremely contagious illness caused by the severe acute respiratory syndrome (SARS-CoV-2) virus. Although this disease primarily involves pulmonary tissue, rapidly advancing research has established cardiac involvement in Covid-19 patients. Objective: This systematic review article aimed to compile and illustrate clinical characteristics, diagnostic findings, management, and outcomes manifesting in myocarditis linked with Covid-19. Methods: A literature search was accomplished for published eligible articles with MEDLINE/PubMed and Embase databases. All eligible case reports and case series were included from around the world without any language restrictions. For this review, inclusion criteria were laboratoryconfirmed SARS-CoV-2 infection cases reporting a diagnosis of acute myocarditis. Results: Data from 41 studies describing myocarditis in 42 Covid-19 patients was obtained. The median age of these patients was 43.4 years, with $71.4 \%$ of them being male. Fever was the most prevalent presenting symptoms seen in $57 \%$ of patients. Hypertension was the most pervasive comorbidity accompanying these patients. Cardiac biomarkers troponin and Brain natriuretic peptide (BNP) were raised in almost $90 \%$ and $87 \%$ of patients, respectively. Electrocardiogram findings were Non-specific and included ST-segment and T-wave changes. The most prevalent histopathological feature appreciated was diffuse lymphocytic inflammatory infiltrates. Antivirals and corticosteroids were the most frequently used medications. About $38 \%$ of patients also needed vasopressor assistance. Out of 42 patients, $67 \%$ recovered, and eight died. Conclusion: Due to the risk of a sudden worsening of patients conditions and myocarditis association with considerable mortality and morbidity, a knowledge of this cardiac complication of Covid-19 disease is crucial for healthcare professionals.
\end{abstract}

Title: Myocarditis associated with Covid-19 disease: a systematic review of published Case reports and Case series

Running head: Myocarditis in SARS-CoV-2 infected patients 
Authors: Sawai Singh Rathore ${ }^{1 *}$, Gianpier Alonzo Rojas ${ }^{2,3}$, Manush Sondhi ${ }^{4}$, Suveenkrishna Pothuru ${ }^{5}$, Reshma Pydi ${ }^{6}$, Neeraj Kancherla ${ }^{7}$, Romil Singh ${ }^{8}$, Noman Khurshid Ahmed ${ }^{9}$, Jill Shah ${ }^{10}$, Sohaib Tousif 11, Unaiza Tariq Baloch ${ }^{12}$, Qingqing Wen ${ }^{13}$

Affiliations: ${ }^{1}$ Internal Medicine, Dr. Sampurnanand Medical College, Jodhpur, India; ${ }^{2}$ Internal Medicine, School of medicine,Universidad Peruana Cayetano Heredia, Lima, Perú ${ }^{3}$ Scientific society of medical students, Cayetano Heredia, Lima, Perú $;{ }^{4}$ Internal Medicine, Kasturba Medical College, Manipal, India ${ }^{5}$ Internal Medicine, Ascension via christi hospital, Manhattan, USA ${ }^{6}$ Internal Medicine, Andhra Medical College, Visakhapatnam, India ${ }^{7}$ Internal Medicine, Andhra Medical College, Visakhapatnam, India ${ }^{8}$ Department of Critical Care, Mayo Clinic, Rochester, USA ${ }^{9}$ Internal Medicine, Dow Medical College, Karachi, Pakistan ${ }^{10}$ Internal Medicine, Tambov state University named after G.R.Derzhavin, Tambov, Russia ${ }^{11}$ Internal Medicine, Ziauddin Medical university, Karachi, Pakistan

${ }^{12}$ Internal Medicine, Aga Khan University Hospital, Karachi, Pakistan ${ }^{13}$ University of California Los Angeles, Fielding School of Public Health, Los Angeles, USACorrespondence : Sawai Singh Rathore, Internal Medicine, Dr. Sampurnanand Medical College, Residency Road, Shastri nagar, Jodhpur, 342003, India E-mail: Sawais.rathore77@gmail.com

\section{ACKNOWLEDGMENTS}

None

\section{Disclosure}

Authors have no potential conflicts of interest to disclose.

\section{Hosted file}

Myocarditis COVID-19 JAN 9.pdf available at https://authorea.com/users/393104/articles/ 506810-myocarditis-associated-with-covid-19-disease-a-systematic-review-of-publishedcase-reports-and-case-series 


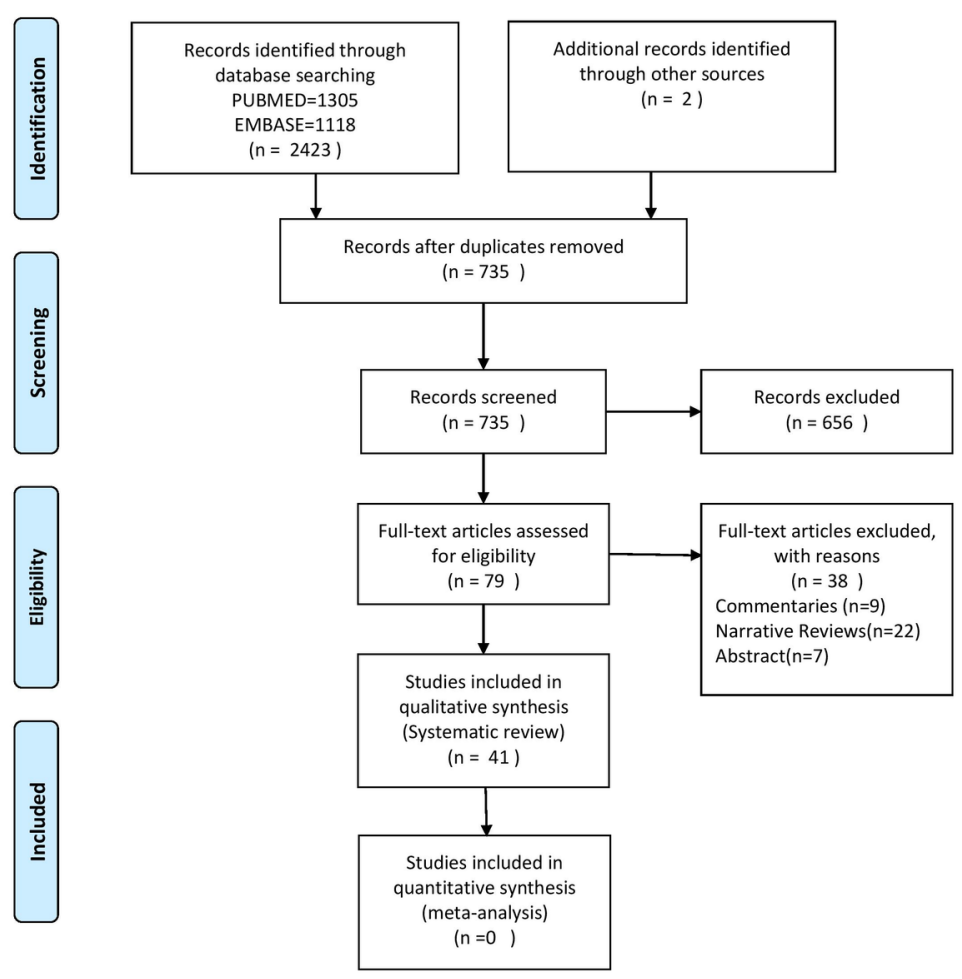

